\title{
Metabolic Requirements of Blood Vessels in a Perfusion Bioreactor
}

\author{
M. Hoenicka ${ }^{1}$, L. Wiedemann ${ }^{2}$, S. Schrammel ${ }^{3}$, C. Schmid ${ }^{1}$, and D.E. Birnbaum ${ }^{1}$ \\ ${ }^{1}$ University of Regensburg Medical Center, Department of Cardiothoracic Surgery, Regensburg, Germany \\ ${ }^{2}$ Klinikum Landshut, Landshut, Germany \\ ${ }^{3}$ University of Applied Sciences, FB Maschinenbau, Regensburg, Germany
}

\begin{abstract}
Small caliber vessel grafts are one of the major challenges of vascular tissue engineering. A variety of processes have been developed to create vascular grafts from scaffolds and donor cells in bioreactors. In order to optimize such processes, this study focused on monitoring vessel metabolism under conditions typically used in perfusion protocols. Bovine veins were perfused in a bioreactor for four days. Group 1 vessels served as controls and were perfused with standard medium. Medium of group 2 was adjusted to the viscosity of blood. Group 3 vessels were additionally challenged with elevated luminal pressure. Contractile function was assessed in an organ bath. Tissue viability was determined by tetrazolium dye reduction. Oxygen gradients, dextrose consumption, and lactate production were monitored using a blood gas analyzer. $\mathrm{KCl}$ induced contractions did not differ between days 0 and 4 . Norepinephrine dose-response curves of group 3 vessels achieved a higher maximum contraction on day 4 , with no changes of $\mathbf{E C}_{50}$. Tissue viability was not altered by any of the perfusion conditions. Oxygen gradients across the vessels did not change with time but were elevated in group 2, with no signs of oxygen depletion. Dextrose consumption and lactate formation of group 1 and 2 vessels appeared to be stoichiometric. In contrast, group 3 vessels produced more lactate than dextrose could supply. These results indicate that conventional oxygenation of culture media is sufficient to meet the oxygen consumption of a functional vessel. Elevated shear forces increased the oxygen demand without affecting dextrose consumption. Elevated shear forces and luminal pressure caused the utilization of alternative energy sources. Thus online monitoring of key metabolic parameters appears to be a desirable feature of perfusion bioreactors for vascular tissue engineering.
\end{abstract}

Keywords - tissue engineering, vascular function, perfusion bioreactor, metabolism

\section{INTRODUCTION}

Coronary artery disease is a common condition in Western countries. Many patients eventually have to undergo coronary artery bypass grafting to restore blood flow in the affected areas of the heart. Autologous vessels like saphenous vein and mammary artery are used as grafts with excellent results. However, an increasing number of patients can- not be treated adequately due to a lack of suitable vessels, usually as a consequence of prior removal, accidents, or diseases which affect the vessel function.

Synthetic polymers turned out to be unsuitable to build small caliber vessel grafts due to their limitations in terms of compliance and surface properties [1]. Instead, various approaches have been suggested to create vessel grafts from natural or biocompatible scaffolds seeded with vascular cells [2]. These tissue engineering procedures usually involve incubations in perfusion bioreactors which provide suitable conditions for graft development. Increased shear forces and/or luminal pressure are often applied in order to adapt the grafts to the conditions of the coronary circulation. To the best of our knowledge, no systematic investigation of the vessel wall metabolism under these conditions has been published so far.

This study presents initial results from a series of experiments designed to describe the vessel wall metabolism in a perfusion bioreactor using bovine veins as a model. Key parameters of the metabolism such as oxygen gradients, dextrose consumption, and lactate production were monitored in order to determine the influences of shear forces and luminal pressure.

\section{Materials AND Methods}

\section{Bovine vein harvesting}

Bovine lateral saphenous veins were harvested from the hindlegs of freshly sacrificed animals and stored in KrebsHenseleit buffer [3]. Veins were dissected free from surrounding tissue in a sterile fashion and side branches were ligated. Samples were taken for measuring tissue viability and vasoconstriction. Segments of $8 \mathrm{~cm}$ length were mounted in vessel chambers.

\section{Perfusion bioreactor}

Vessel chambers were attached to custom-built perfusion bioreactors operated at $37^{\circ} \mathrm{C}$. Each circulation consisted of medium reservoir, hollow fiber oxygenator $\left(20 \% \mathrm{O}_{2}, 5 \%\right.$ 
$\mathrm{CO}_{2}$ ), perfusion and superfusion circuit pump hoses, compliance chambers, pressure probes, and Starling resistors. All circulations shared one perfusion and one superfusion peristaltic pump. Ports upstream and downstream of the vessel chambers allowed to retrieve medium samples in a sterile fashion. Perfusion and superfusion pumps were calibrated to deliver $40 \mathrm{ml} / \mathrm{min}$ and $20 \mathrm{ml} / \mathrm{min}$, respectively.

\section{Perfusion conditions}

The perfusion medium was M199 with Earle's salts supplemented with $20 \%$ fetal calf serum, penicillin, streptomycin, amphotericin B, and gentamicin. The viscosity was increased to that of blood in some experiments. To this end, $12 \%(\mathrm{w} / \mathrm{v})$ dextran (average molecular weight 40000$)$ were added to the default medium. The viscosity was verified in a rotational rheometer. The duration of the perfusion experiments was 4 days, with one medium change. The perfusion conditions used in this study are summarized in Table 1.

Table 1 Perfusion conditions

\begin{tabular}{llll}
\hline \multicolumn{1}{c}{ group } & 1 & 2 & 3 \\
\hline Number of animals & 16 & 5 & 8 \\
dextran & none & none & $12 \%(\mathrm{w} / \mathrm{v})$ \\
pressure & none & none & Luminal $43 \mathrm{~mm} \mathrm{Hg}$ \\
flow & laminar & laminar & pulsatile \\
\hline
\end{tabular}

\section{Determination and analysis of key metabolic parameters}

Medium samples were retrieved daily upstream and downstream of the vessel chambers and analyzed in a blood gas machine. The differences in oxygen partial pressures upstream and downstream of the vessels were assumed to be proportional to the oxygen consumption. Dextrose consumption and lactate production were compared under the assumption of a stoichiometric conversion (anaerobic glycolysis). To test this hypothesis, the glycolytic index was calculated according to equation 1 :

$$
\text { glycolytic index }=\frac{\text { cumulative } \Delta \text { [lactate] }}{(-2) \cdot \text { cumulative } \Delta \text { [dextrose] }}(1)
$$

Values close to unity indicate stoichiometric conversion of dextrose to lactate. Lower values indicate oxidative phosphorylation, whereas higher values indicate the usage of other energy sources.

\section{Contractile properties}

Contractile functions of vessels were determined isometrically in an organ bath as described previously [3]. Maximum contractions caused by depolarization were determined by adding $150 \mathrm{mM} \mathrm{KCl}$ to the baths. Receptor-dependent responses to noradrenaline were evaluated by constructing cumulative dose-response curves.

\section{Determination of tissue viability}

Tissue viability was assessed by measuring the capacity to reduce the tetrazolium compound 3-(4,5-dimethylthiazol2-yl)-5-(3-carboxymethoxyphenyl)-2-(4-sulfophenyl)-2Htetrazolium (MTS) to a coloured formazan [4]. Vessel segments were opened longitudinally and inserted into custombuilt chambers which expose $0.30 \mathrm{~cm}^{2}$ of the luminal surface to the reagent. The dye conversion was then determined according to the manufacturer's instructions .

\section{RESULTS}

\section{Shear stress}

Shear stress in human veins in vivo is in the range of 1 to $6 \mathrm{dyn} / \mathrm{cm}^{2}$ [5]. The internal diameter of the veins used in this study was approx. $5 \mathrm{~mm}$. Addition of dextran increased the viscosity of the medium from $75 \pm 1 \mathrm{mPa} \cdot \mathrm{s}$ to $270 \pm 4$ $\mathrm{mPa} \cdot \mathrm{s}$. The resulting wall shear stresses were computed as $0.4 \mathrm{dyn} / \mathrm{cm}^{2}$ and $1.5 \mathrm{dyn} / \mathrm{cm}^{2}$, respectively, which puts the adjusted medium in the lower physiological range.

\section{Influence of perfusion on contractile function and on tissue viability}

The maximum contractions induced by depolarization with $\mathrm{KCl}$ did not differ between days 0 and 4 (Table 2).

Table $2 \mathrm{KCl}$ induced contractions $(\mathrm{mN})$

\begin{tabular}{llll}
\hline \multicolumn{1}{c}{ group } & 1 & 2 & 3 \\
\hline day 0 & $35.9 \pm 19.8$ & $24.6 \pm 17.1$ & $33.0 \pm 22.4$ \\
day 4 & $32.2 \pm 17.3$ & $35.4 \pm 15.6$ & $45.1 \pm 17.5$ \\
& $\mathrm{p}=0.572$ & $\mathrm{p}=0.556$ & $\mathrm{p}=0.332$ \\
\hline
\end{tabular}

Noradrenaline dose-response curves did not differ significantly between days 0 and 4 in groups $1(p=0.655), 2$ $(\mathrm{p}=0.370)$, and $3(\mathrm{p}=0.283$, ANOVA, Fig. 1). However, group 3 vessels responded significantly stronger to noradrenaline compared to the other groups on day $4(p<0.001)$. $\mathrm{EC}_{50}$ values did not differ between groups $(\mathrm{p}=0.208$, ANOVA).

The viability of the luminal faces of the vessels as determined by their ability to reduce MTS was not influenced by the perfusion conditions (Table 3 ). 


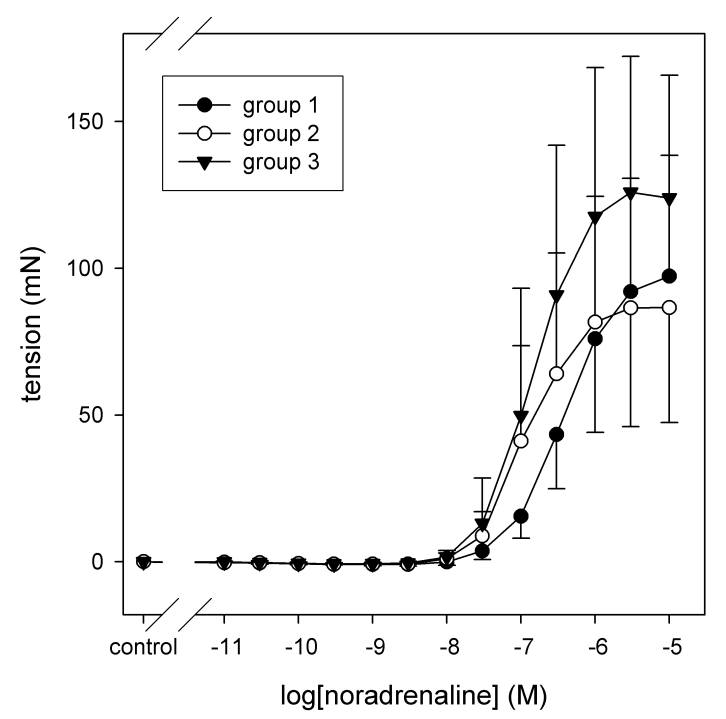

Figure 1: Noradrenaline dose-response curves of bovine saphenous veins perfused for 4 days. Group 3 vessels contracted significantly stronger compared to the other groups $(\mathrm{p}<0.001$, ANOVA)

Table 3 Tetrazolium dye reduction $\left(\mathrm{OD}_{430}\right)$

\begin{tabular}{llll}
\hline group & 1 & 2 & 3 \\
\hline day 0 & $0.80 \pm 0.25$ & $0.73 \pm 0.25$ & $0.97 \pm 0.20$ \\
day 4 & $0.99 \pm 0.22$ & $1.09 \pm 0.14$ & $0.98 \pm 0.21$ \\
& $\mathrm{p}=0.068$ & $\mathrm{p}=0.333$ & $\mathrm{p}=0.946$ \\
\hline
\end{tabular}

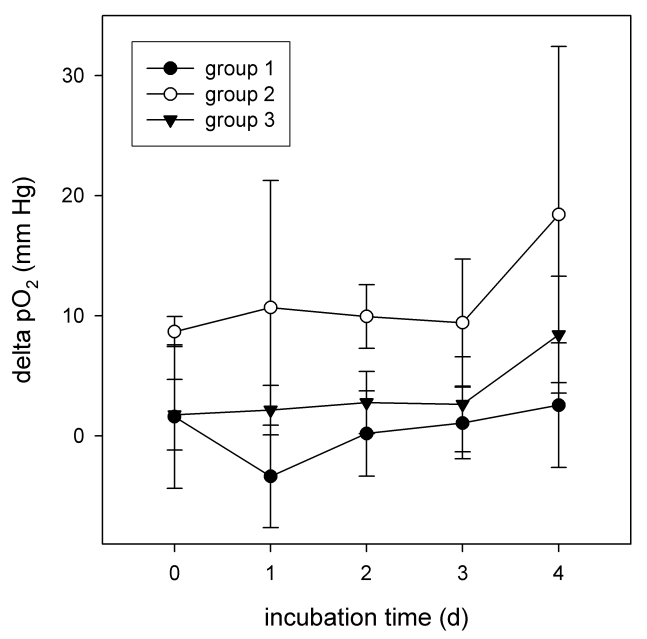

Figure 2: Oxygen partial pressure (pO2) gradient between inletand outlet of he vessel chamber. The gradient was significantly higher in group 2 $(\mathrm{p}<0.001$, ANOVA) but there wereno significant changes over time

\section{Changes of oxygen partial pressures}

The average oxygen partial pressures at the vessel chamber inlets was $147.4 \pm 2.4 \mathrm{~mm} \mathrm{Hg}$. There was no correlation with perfusion conditions or time. The oxygen gradients between vessel chamber inlets and outlets were different between perfusion groups (Fig. 2, $\mathrm{p}<0.001$, ANOVA), indicating an increased oxygen consumption in group 2.

\section{Changes of lactate and dextrose concentrations}

There was a linear decrease of dextrose concentrations and a linear increase of lactate concentrations over time in all perfusion groups (Table 4). Vessels of group 3 showed a glycolytic index significantly higher than unity.

Table 4 Dextrose consumption and lactate production

\begin{tabular}{llll}
\hline \multicolumn{1}{c}{ group } & 1 & 2 & 3 \\
\hline $\begin{array}{l}\text { dextrose consump- } \\
\text { tion (mM/d) }\end{array}$ & 0.51 & 0.68 & 0.43 \\
$\begin{array}{l}\text { lactate production } \\
\text { (mM/d) }\end{array}$ & 1.18 & 1.38 & 1.36 \\
glycolytic index & 1.16 & 1.01 & $1.58^{* * *}$ \\
& n.s. & n.s. & $* * * \mathrm{p}<0.001$ \\
\hline
\end{tabular}

\section{DisCUSSION}

This study used bovine veins in order to determine whether standard culture media and hollow fiber oxygenators are capable of sustaining the function and viability of vessels under flow and pressure conditions resembling those used in vascular tissue engineering protocols.

M199 contains dextrose at $5.6 \mathrm{mmol} / \mathrm{l}$ as the major energy source. Most cells prefer to utilize external dextrose over using intracellular sources like glycogen. [6]. The analysis of cumulative dextrose consumption and lactate formation rates in our study clearly indicate a prevalence of lactate fermentation in the absence of luminal pressure. One mol of dextrose yields two mol of lactate, with a concomitant formation of 2 adenosine triphosphate (ATP) units. These data confirm earlier findings obtained in vascular tissue slices or organ bath preparations [7]. This is in stark contrast to skeletal muscle which uses this type of lactic acid fermentation only under hypoxia. Given a sufficient oxygen supply, oxidative phosphorylation in the mitochondria of skeletal muscle cells provides approx. another 30 ATP. The results of this study indicate that circumferential stress, most of which is compensated by smooth muscle contraction, does not induce oxidative phosphorylation in vascular smooth muscle cells (VSMC), although the latter were reported to have a normal mitochondrial mass and 
function [7]. This highly economical muscle function appears to be a specific feature of slow, tonic smooth muscle cells as opposed to fast and spontaneously contracting smooth muscles. Under circumferential stress, other substrates besides dextrose seem to be utilized. Several alternative energy sources are available in the culture medium, e.g. L-glutamine, L-leucine, and L-valine, all of which can be utilized for energy production by VSMC [8;9]. Rabbit artery VSMC increased glucose and glutamine uptake as well as lactate production after electrical stimulation [10]. It may be prudent to monitor the concentrations of alternative substrates in the medium and replenish them if required.

As a consequence of the lack of oxidative phosphorylation during dextrose breakdown, oxygen partial pressure was obviously not limiting vessel function. This is clearly shown by the oxygen partial pressure of the vessel efflux which does not even come close to hypoxia. Therefore, standard methods like gassing the media with $20 \%$ oxygen are sufficient to maintain vessel function.

The contractile function of the vessels remained intact in our perfusion system for at least 4 days. Contractions induced by $\mathrm{KCl}$ were identical in all groups before and after perfusion. However, vessels of group 3 responded stronger to noradrenaline after perfusion compared to the other groups, demonstrating a benefit of circumferential stress in a perfusion bioreactor. The amount of tetrazolium dye reduction as an indicator of tissue viability [11] did not change within 4 days, indicating that the nutrition and the perfusion conditions are suitable.

Some limitations need to be addressed in future studies. The consumption of other energy sources should be monitored by analyzing the spent medium to further identify the major sources of energy under load. Four days are arguably too short to provide data comparable to tissue-engineering protocols which often take several weeks to complete. Finally, only equivalent experiments using seeded scaffolds to test the oxygen and nutrient consumption of growing cells will provide more specific information in order to optimize vascular tissue engineering processes.

In conclusion, the results of the present study indicate that metabolism should be monitored closely in all vascular tissue engineering projects as it reflects the perfusion conditions under which the vessel graft is developed. Vessel wall metabolism is apparently not limited by the oxygen supply provided by aqueous media at $20 \%$ oxygen which permits application of partial pressures which best stimulate growth of varous vascular cell types. As dextrose is apparently not the only energy source of vessels under load, tissue culture media may have to be adapted to supply sufficient amounts of alternative substrates and to decrease lactate production in order to avoid costly media changes during the production of tissue-engineered vessel grafts.

\section{ACKNOWLEDGMENT}

This study was supported by Deutsche Forschungsgemeinschaft (BI-139/2-1).

\section{REFERENCES}

1. Hoenig MR, Campbell GR, Campbell JH (2006) Vascular grafts and the endothelium. Endothelium 13:385-401

2. Yow K, Ingram J, Korossis SA, et al. (2006) Tissue engineering of vascular conduits. Br J Surg 93:652-661

3. Hoenicka M, Lehle K, Jacobs VR, et al. (2007) Properties of the human umbilical vein as a living scaffold for a tissue-engineered vessel graft. Tissue Eng 13:219-229

4. Berridge MV, Herst PM, Tan AS (2005) Tetrazolium dyes as tools in cell biology: new insights into their cellular reduction. Biotechnol Annu Rev 11:127-152

5. Paszkowiak JJ, Dardik A (2003) Arterial wall shear stress: observations from the bench to the bedside. Vasc Endovascular Surg 37:47-57

6. Paul RJ, Krisanda JM, Lynch RM (1984) Vascular smooth muscle energetics. J Cardiovasc Pharmacol 6 Suppl 2:S320-S327

7. Paul RJ (1980) Chemical energetics of vascular smooth muscle. In: Bohr DF, Somlyo AP, Sparks HVJ. Vascular smooth muscle. American Physiology Society, Bethesda, MD, pp. 201-235

8. Chace KV, Odessey R (1981) The utilization by rabbit aorta of carbohydrates, fatty acids, ketone bodies, and amino acids as substrates for energy production. Circ Res 48:850-858

9. Glacken MW, Fleischaker RJ, Sinskey AJ (1986) Reduction of waste product excretion via nutrient control: Possible strategies for maximizing product and cell yields on serum in cultures of mammalian cells. Biotechnol Bioeng 28:1376-1389

10. Heinle H, Sigg G, Reich A, et al. (1984) Metabolic effects of direct current stimulation on cultured vascular smooth muscle cells. Z Naturforsch [C] 39:1141-1144

11. Marshall NJ, Goodwin CJ, Holt SJ (1995) A critical assessment of the use of microculture tetrazolium assays to measure cell growth and function. Growth Regul 5:69-84

Author: Markus Hoenicka, $\mathrm{PhD}$

Institute: University of Regensburg Medical Center, Department of Cardiothoracic Surgery

Street: Franz-Josef-Strauss-Allee 11

City: 93053 Regensburg

Country: Germany

Email: markus.hoenicka@klinik.uni-regensburg.de 